\title{
Therapeutic Intervention Scoring System in Surgical Intensive Care (Our Expirience)
}

\author{
Ljiljana Gvozdenovic $^{{ }^{*}}$, Mirka Lukic ${ }^{2}$, Teodora Bozic ${ }^{3}$, Natasa Nestorov ${ }^{4}$ \\ Medical University, School of Medicine, Clinical center Vojvodina, Novi Sad, Serbia
}

*Corresponding Author: Ljiljana Gvozdenovic, Clinical Center of Vojvodina, Novi Sad, Serbia, Email: ljiljana.gvozdenovic@mf.uns.ac.rs

\section{SHORT COMMUNICATION}

The aim of this study was to estimate the quantitative / numerical measurements using TISS-28 scale, objectively demonstrate how the work of nurses / technicians employ the most difficult patients (APACHE II score> 25) in the Department of Surgical Intensive Care (ICU),Department of Anesthesiology and Intensive Care, Clinical Center, Novi Sad, Serbia, in 2016. The experiment was conducted retrospective study 595 adult patients of both sexes, who were treated in the surgical ICU were hospitalized for more than 24 hours, and the value of APACHE II score on admission to them was higher than the $25^{\text {th }}$. The average occupancy in 2011. The year was $110 \%$ (minimum $88 \%$, maximum $124 \%$ ). In $90 \%$ of hospitalized patients was applied mechanical lung ventilation and invasive monitoring. Nurses working in two shifts of 12 hours, the nurse ratio 1:2 patient per shift. Doctors have a 24-hour duty (a doctor) during regular business hours there is another doctor and head of the department that it's full-time spent in ICU. In addition to the study of demographic data of the patients was recorded as the value of TISS- 28 for each patient on admission and every nursing shift and APACHE II score at admission and during hospitalization in the ICU. Recorded the length of hospitalization in the ICU outcome.

All data are presented in tables and graphs using Microsoft Excel. Statistical processing of the results was performed by basic descriptive methods: the mean and standard deviation for numerical parameters. From the existence of statistically significant differences of parametric data was tested Student $t$ test. All $p$ values $<0.05$ were considered statistically significant.
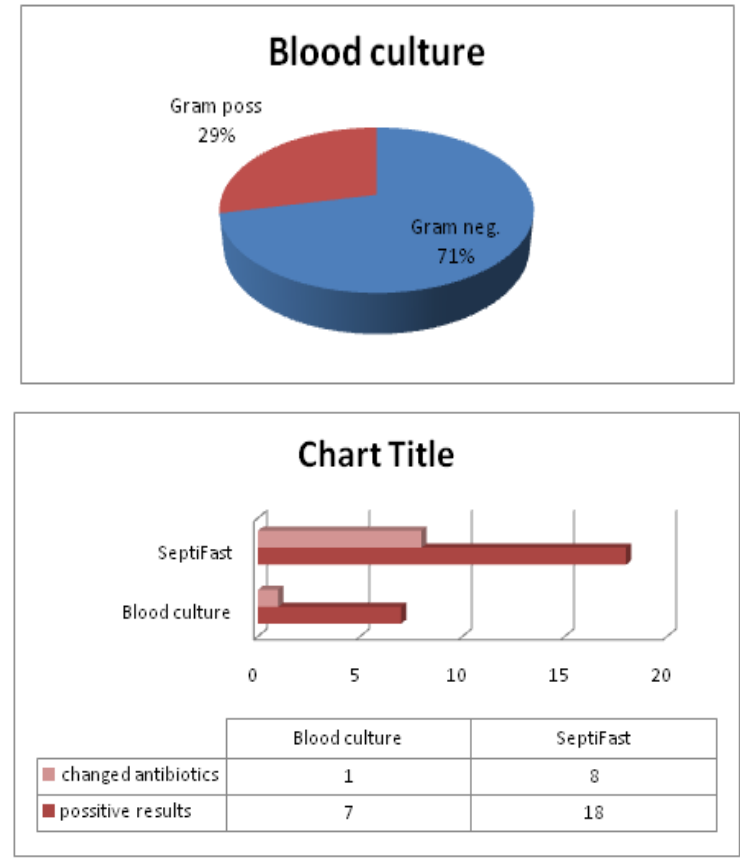

In the study patients was 380 male. Ages ranged from 18 to 82 years. The average age was 58.92 \pm 15.50 years. All the patients on admission to the ICU had a value of APACHE II score> 25. The mean value of TISS on day of admission was $37.02 \pm 6.90$ points. The average length of stay in the ICU-in was $9.49 \pm 7.56$ days. In the class I and II ( 0 to 9 and 10 to 19 TISS points per shift) was not a patient. In class III (20-39 points) were 570 patients, in class IV (over 40 points) was 380 patients. The average age of the patients were in class III according to TISS-in was $55.52 \pm 16.13$ years, while those in class IV according to TISS-in was $62.53 \pm 13.91$. A statistically significant difference in age who were in class III and IV at TISS-in. The average length of stay in the ICU-patients who were in class III at TISS-in was $7.99 \pm 6.70$ days. The average length of stay in the ICU-who were in class IV at TISS-in was $11.73 \pm 8.11$ days. There was a statistically significant difference in 
the length of hospitalization in ICU patients, Class III and Class IV the values TISS score.

In our study, the mean TISS score on the day of admission was higher than in the studies because the study included patients with APACHE II scores> $25(1,2)$. It should be noted that the value of TISS score also depends on the level of occupancy and the number of beds I ICU $(3,4)$. With this in mind the lower TISS could be an expression of ICU larger capacity, making it possible for the greater number of patients in the short-term monitoring and treatment, and patients in better general condition (5).

As a nurse with experience can take care of a single patient who has a 40-50 TISS points. For this class IV requires 1:1 ratio patient: nurse, but sometimes may require additional assistance. As in the study ICU nurse ratio: 1:2 patient, it can be concluded that the number of nurses / technicians were insufficient. The obtained high values of TISS-score for severely ill patients suggest that the nurses working in ICU, very hard and are insufficient. The results would certainly be more convincing if they were made for a longer period of time and for all patients.

\section{REFERENCES}

[1] Cheng B, Xie G, Yao S, Wu X, Guo Q, Gu M, Fang Q, Xu Q, Wang D, Jin Y, Yuan S, Wang J, Du Z, Sun Y, Fang X. Epidemiology of severe sepsis in critically ill surgical patients in ten university hospitals in China. Crit Care Med. 2007;35:2538-2546.ž

[2] Muehler N, Oishi J, Specht M, Rissner F, Reinhart K, Sakr Y. Serial measurement of Therapeutic Intervention Scoring System-28 (TISS-28) in a surgical intensive care unit. J Crit Care. 2010 Dec; 25(4):620-7.

[3] Lefering R, Zart M, Neugebauer EA. Retrospective evaluation of the simplified Therapeutic Intervention Scoring System (TISS-28) in a surgical intensive care unit. Intensive Care Med. 2000 Dec;26(12):1794802.

[4] Panagiotis Kiekkas, George C. Sakellaropoulos, Hero Brokalaki, Evangelos Manolis, Adamantios Samios, Chrisula Skartsani, George Baltopoulos Association Between Nursing Workload and Mortality of Intensive Care Unit Patients. JOURNAL OF NURSING SCHOLARSHIP, 2008; 40:4, 385-390.

[5] Vasquez DN, Estenssoro E, Canales HS. Clinical characteristics and outcomes of obstetric patients requiring ICU admission. Chest. 2007; 131: 718-724.

Citation: Ljiljana Gvozdenovic. Therapeutic Intervention Scoring System in Surgical Intensive Care (Our Expirience). ARC Journal of Anesthesiology. 2018; 3(1):1-2. Doi: dx.doi.org/10.20431/2455-9792.0301001.

Copyright: () 2018 Authors. This is an open-access article distributed under the terms of the Creative Commons Attribution License, which permits unrestricted use, distribution, and reproduction in any medium, provided the original author and source are credited. 